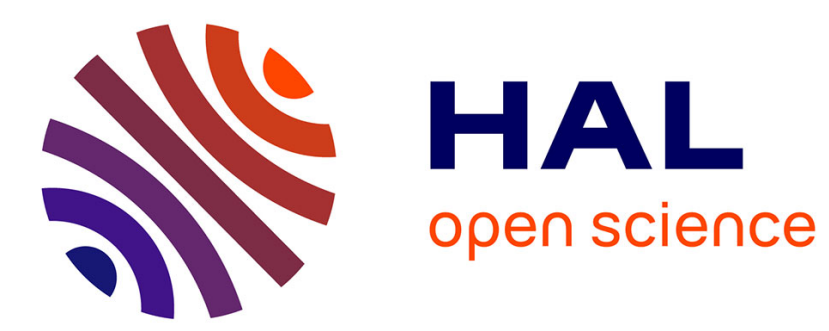

\title{
Influence de la chimisorption sur le courant d'obscurité du CdS de résistivité élevée
}

S. Ravelet, W. Palz

\section{To cite this version:}

S. Ravelet, W. Palz. Influence de la chimisorption sur le courant d'obscurité du CdS de résistivité élevée. Revue de Physique Appliquée, 1970, 5 (2), pp.321-326. 10.1051/rphysap:0197000502032100 . jpa-00243395

\section{HAL Id: jpa-00243395 https://hal.science/jpa-00243395}

Submitted on 1 Jan 1970

HAL is a multi-disciplinary open access archive for the deposit and dissemination of scientific research documents, whether they are published or not. The documents may come from teaching and research institutions in France or abroad, or from public or private research centers.
L'archive ouverte pluridisciplinaire HAL, est destinée au dépôt et à la diffusion de documents scientifiques de niveau recherche, publiés ou non, émanant des établissements d'enseignement et de recherche français ou étrangers, des laboratoires publics ou privés. 


\title{
INFLUENCE DE LA CHIMISORPTION SUR LE COURANT D'OBSCURITÉ DU CdS DE RÉSISTIVITÉ ÉLEVÉE
}

\author{
par S. RAVELET $\left({ }^{1}\right)$ et W. PALZ, \\ Laboratoire d'Electronique et de Physique du Solide, E. N. S. E. M., 2, rue de la Citadelle, Nancy
}

(Reçu le 20 octobre 1969, revisé le $1^{\text {er }}$ décembre 1969)

\begin{abstract}
Résumé. - Nous avons mesuré la conductivité à l'obscurité de monocristaux très minces de CdS en fonction de l'état de chimisorption d'oxygène à la surface. Les études effectuées avec différents contacts montrent que la conductivité en volume est affectée par les niveaux de surface. On conclut que tous les porteurs du volume sont piégés par les niveaux de surface. Sous oxygène et sous vide, la conductivité, dans l'état adsorbé, est faible $\left(10^{-15}(\mathrm{ohm} . \mathrm{cm})^{-1}\right)$. L'augmentation de la conductivité $\left(5 \times 10^{-9}(\mathrm{ohm} . \mathrm{cm})^{-1}\right)$ après bombardement électronique est interprétée comme une désorption d'oxygène. Sur un cristal adsorbé (conductivité faible), la mesure de la conductivité en fonction de la température montre l'existence de niveaux situés à $0,4 \mathrm{et} 0,9 \mathrm{eV}$ en dessous de la bande de conduction. Ceux-ci sont attribués à deux états d'ionisation de l'oxygène à la surface du $\mathrm{CdS}$, appelés ( $\mathrm{O}^{--}$) et ( $\mathrm{O}^{-}$). La même mesure effectuée sur un cristal (( désorbé )) (conductivité élevée) permet d'atteindre des niveaux situés à 0,04, 0,06 et 0,16 eV en dessous de la bande de conduction. En comparant les effets d'un bombardement électronique $(6 \mathrm{keV})$ d'une part et d'une irradiation lumineuse (4 $500 \AA$ A) d'autre part, sur la conductivité à l'obscurité, on conclut que la désorption par trous libres est relativement inefficace.

Lorsque le cristal est irradié par des électrons $\left(5 \times 10^{-6} \mathrm{~A} / \mathrm{cm}^{2}\right)$, la conductivité à l'obscurité augmente en fonction de la durée $t$ de l'exposition suivant une loi en $t^{1,7}$. On peut déduire que la désorption est un processus qui transforme l'oxygène se trouvant dans un certain état de chimisorption $\left(\mathrm{O}^{--}\right)$en oxygène physisorbé par l'intermédiaire d'un deuxième état de chimisorption $\left(\mathrm{O}^{-}\right)$. Une dose de $10^{17}$ électrons $/ \mathrm{cm}^{2}$ est nécessaire pour la désorption optimale.
\end{abstract}

\begin{abstract}
The influence of chemisorption on the dark current has been studied on thin CdS platelets. Measurements with different contacts showed that the bulk conductivity was affected by surface adsorption. It was concluded that all volume carriers were trapped on the surface. The conductivity of the adsorbed platelets was $10^{-15}(\mathrm{ohm} . \mathrm{cm})^{-1}$ in an oxygen atmosphere as well as in vacuum. The enhancement of conductivity $\left(5 \times 10^{-9}(\mathrm{ohm} . \mathrm{cm})^{-1}\right)$ after an electron bombardment can be attributed to oxygen desorption. Energy levels at 0.4 and $0.9 \mathrm{eV}$ were found by measuring the temperature dependence of the conductivity of adsorbed samples in vacuum. The energies were attributed to two different states of ionization of oxygen at the CdS surface called ( $\mathrm{O}^{--}$)) and $\left(\left(\mathrm{O}^{-}\right)\right.$). Bulk donor levels situated at $0.04,0.06$, and $0.16 \mathrm{eV}$ appeared on an oxygen free sample. Desorption by electron bombardment $(6 \mathrm{keV})$ was much more effective than irradiation with intrinsicly absorbed light (4 $500 \AA)$. Thereby it could be concluded that the desorption by recombination of free holes with chemisorbed oxygen is relatively unimportant.

Owing to an bombardment by electrons $\left(5 \times 10^{-6} \mathrm{~A} / \mathrm{cm}^{2}\right)$, the dark current increased with time, following a law $t^{1,7}$. Therefore desorption by electrons should be a two step process : before being neutralized, the chemisorbed oxygen changes its state of ionisation $\left(\mathrm{O}^{--} \rightarrow \mathrm{O}^{-}\right)$. Complete desorption was obtained for a dose of $10^{17}$ electrons $/ \mathrm{cm}^{2}$.
\end{abstract}

Introduction. - Une molécule ou un atome adsorbé à la surface d'un semi-conducteur ou d'un photoconducteur peut agir comme donneur, accepteur ou centre de recombinaison. Cette impureté adsorbée par chimisorption est dans la plupart des cas l'oxygène, mais aussi la vapeur d'eau. Une influence des états de surface sur les caractéristiques électriques a été observée sur le CdS, par exemple sur une structure métal-

(1) Ce travail fait partie d'une thèse qui sera soumise par Mr. S. Ravelet à la Faculté des Sciences de Nancy en vue de l'obtention du Doctorat d'Etat ès Sciences Physiques en 1970. Numéro d'enregistrement au C. N. R. S. : AO 3393. isolant-semiconducteur [1, 2] sur certaines jonctions [3] et sur des piles solaires en couches minces [4, 5]. L'adsorption modifie également le travail de sortie mesuré sur des diodes Schottky au CdS [6]. Par ailleurs, il semble qu'elle joue un certain rôle dans la cathodoluminescence observée sur des cristaux de Cds [7].

De nombreuses études ont d'abord été réalisées dans ce domaine à partir de 1948 sur le $\mathrm{ZnO}$ [8-11] ; aussi un premier modèle physique de la chimisorption a-t-il pu être développé sur ce matériau [12-15]. Ce modèle a été appliqué plus tard au CdS par Williams [16] et par Mark [17, 18]. La plupart des obser- 
vations faites jusqu'ici sur le CdS (références citées en [19]) ont été interprétées à l'aide de ce modèle. Cependant, bien que celui-ci soit bien établi, ces mêmes phénomènes tels que «l'overshoot» du photocourant ou la variation du courant thermiquement stimulé avec la lumière excitatrice ont été attribués à un effet photochimique de volume [20-24].

Nous nous sommes proposés dans ce travail d'étudier l'influence de l'oxygène sur le courant d'obscurité mesuré sur des monocristaux de CdS ayant une surface naturelle. Celle-ci est balayée par un faisceau d'électrons de $6 \mathrm{keV}$ capable de désorber l'oxygène chimisorbé après un séjour dans l'air ou sous oxygène pur. Cette méthode a été choisie en dépit des problèmes qu'elle entraîne à savoir une éventuelle formation de défauts dans le CdS. Il est possible, au cours des cycles d'adsorption et de désorption, de mesurer les variations du courant d'obscurité en fonction de différents paramètres (temps, température, excitation électronique et lumineuse). La détermination des niveaux d'énergie, sur un cristal se trouvant dans l'état adsorbé, donne l'énergie de l'oxygène chimisorbé à la surface, dans l'état désorbé, elle donne l'énergie des donneurs peu profonds.

Dispositif expérimental. - Le cristal de CdS est collé sur un support en verre, il est placé dans une cellule de mesure évacuée à $3 \times 10^{-6}$ torr. Cette cellule est équipée (Fig. 1) :

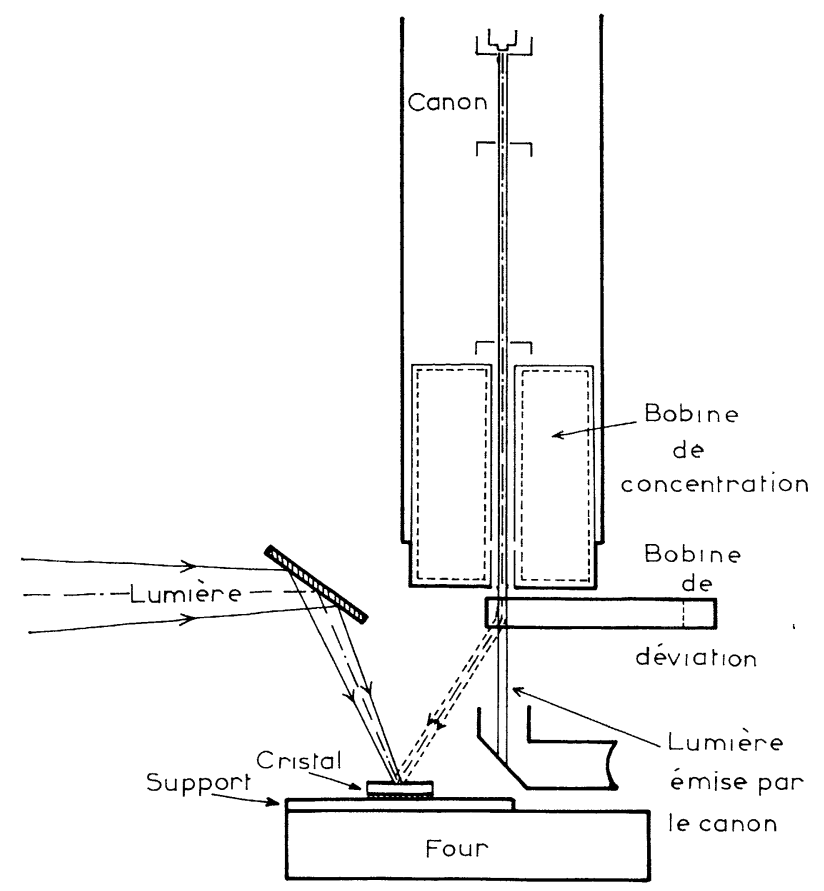

FIG. 1. - Dispositif permettant l'irradiation d'un cristal de CdS par des électrons et par la lumière venant d'un monochromateur. Les électrons sont déviés par un champ magnétique. Experimental arrangement showing the crystal, the electron beam deflected by a magnetic field and the monochromatic light beam.
- d'un four en cuivre avec lequel le cristal est en contact thermique; la température du four peut varier de $77^{\circ} \mathrm{K}$ à $400{ }^{\circ} \mathrm{K}$;

- d'un canon à électrons « CSF démontable HT » qui est utilisé avec une tension d'accélération de $6 \mathrm{kV}$ pour obtenir un courant continu dans la gamme $10^{-12}$ à $10^{-7} \mathrm{~A}$;

- d'un passage optique permettant d'éclairer l'échantillon avec un faisceau de lumière issu d'un monochromateur Bausch et Lomb Haute Intensité équipé d'une source quartz-iode de $150 \mathrm{~W}$. Le faisceau lumineux et le faisceau électronique tombant sur le cristal forment entre eux un angle de $60^{\circ}$. Le flux d'électrons, après concentration par une bobine, passe dans l'entrefer d'un électroaimant et y subit une déviation. Cette déviation possède plusieurs avantages : les ions négatifs n'atteignent pas la surface du cristal, la lumière émise par la cathode tombant à côté de l'échantillon est adsorbée dans une boîte, le faisceau d'électrons peut facilement être réglé sur le cristal. De plus, une commande mécanique extérieure permet de varier l'inclinaison du canon par rapport à la normale au cristal. La cellule de mesure possède une fuite réglable alimentée par un gaz pur déshydraté après circulation dans un piège réfrigéré.

Nos cristaux de CdS ayant $30 \mu \mathrm{m}$ d'épaisseur ont poussé en phase vapeur sous argon et ne sont pas volontairement dopés. Ils sont utilisés sans aucun traitement physique ou chimique, avec leur surface naturelle. Deux électrodes d'indium sont évaporées sur la surface exposée au flux d'irradiation comme l'indique la figure $2 a$. Le cercle pointillé représente l'impact du faisceau électronique de surface $1 \mathrm{~mm}^{2}$. Une troisième électrode est évaporée sur la surface inférieure du cristal. Les courants peuvent être mesurés en superficiel ou en sandwich (Fig. 2b) avec un électromètre Intertechnique A CV 101 vibron.

(a)

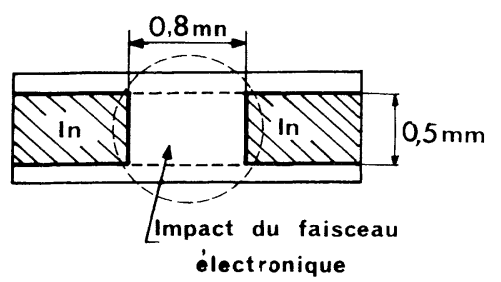

(b)

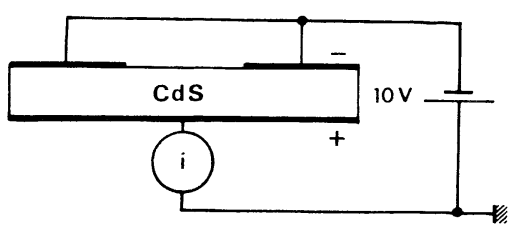

FIG. 2. - Disposition des contacts en Indium sur le cristal. Contact disposition on the Crystal.

Mesures. - Sauf indication contraire, les courants sont mesurés sous vide et en sandwich, le contact 
supérieur étant porté à un potentiel de $-10 \mathrm{~V}$ par rapport au contact inférieur.

- Courant induit dans le CRISTAl PAR bombarDEMENT ÉLECTRONIQUE. - Lorsqu'un cristal ayant séjourné dans l'air est placé sous vide, le courant d'obscurité $i_{0}$ est très faible à l'équilibre thermique $\left(5 \times 10^{-14} \mathrm{~A}\right)$ avant le bombardement électronique représenté sur la figure 3 (encart). Sur cette dernière, les courbes a et $b$ représentent les courants induits dans le cristal par bombardement électronique en fonction des intensités d'excitation respectivement croissantes et décroissantes. La courbe $b$ peut, après avoir été parcourue une première fois dans le sens de la flèche, être parcourue dans les deux sens de façon reproductible si le cristal reste sous vide. Si ce dernier est exposé à l'air, le courant induit par bombardement électronique suit à nouveau la courbe a ou une courbe intermédiaire entre $a$ et $b$ selon la durée et les conditions de cette exposition. Des phénomènes semblables ont été décrits récemment dans la littérature $[25,26]$.

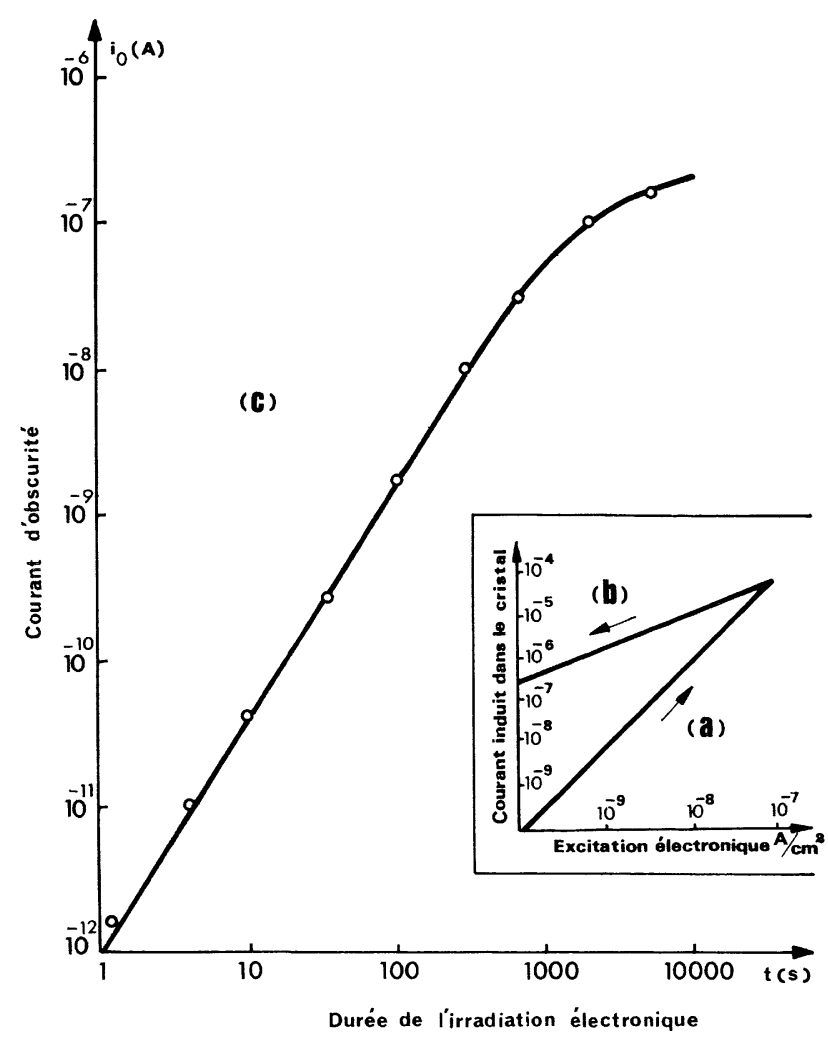

Fig. 3. - $a$ ) et $b$ ) Courant induit sur un cristal non traité en fonction de l'intensité de l'excitation électronique. c) Courant d'obscurité mesuré en fonction de la durée de l'exposition à un faisceau électronique de $5 \times 10^{-6} \mathrm{~A} / \mathrm{cm}^{2}$. Après chaque point de mesure le cristal est de nouveau exposé à l'oxygène

$$
\left(i_{0}=5 \times 10^{-14} \mathrm{~A}\right) \text {. }
$$

$a$ ) and $b$ ) Current induced by electron bombardment on a ( as grown) crystal versus bombardment intensity. c) Dark current versus exposition time to an electron bombardment of $5 \times 10^{-6} \mathrm{~A} / \mathrm{cm}^{2}$. After every point the crystal is re-exposed to oxygen $\left(i_{0}=5 \times 10^{-14} \mathrm{~A}\right)$.
- VARIATION DU COURANT D'OBSCURITÉ EN FONCTION DE LA DURÉE DU BOMBARDEMENT ÉLECTRONIQUE - Le courant d'obscurité est mesuré sous vide à $20{ }^{\circ} \mathrm{C}, 12$ heures après la dernière excitation ; pendant ces 12 heures, le cristal subit plusieurs cycles d'irradiation infrarouge (quenching à $1,4 \mu \mathrm{m}$ ) séparés par des périodes d'obscurité, sinon, il faudrait plusieurs semaines pour atteindre l'équilibre thermique. Le courant d'obscurité mesuré dans ces conditions, après avoir terminé le cycle $a-b$, est de l'ordre de $10^{-9} \mathrm{~A}$ au lieu de $5 \times 10^{-14} \mathrm{~A}$ avant le cycle. Sa valeur, après excitation électronique, dépend de l'intensité et de la durée de cette dernière. Le courant d'obscurité mesuré après le bombardement du cristal par un flux d'électrons de $5 \times 10^{-6} \mathrm{~A} / \mathrm{cm}^{2}$ est représenté sur la courbe $c$ (Fig. 3) en fonction de la durée de cette irradiation ; avant chaque exposition au bombardement électronique, le cristal se trouve dans un état d'adsorption bien déterminé ; celui-ci est défini par un courant d'obscurité de $5 \times 10^{-14} \mathrm{~A}$ mesuré sous vide ; il est atteint en exposant l'échantillon à l'oxygène sec pendant une heure, entre chaque point de mesure. Pendant cette exposition, l'échantillon est éclairé $(\lambda=5150 \AA)$. Le courant d'obscurité $i_{0}$ croît avec la durée $t$ de l'irradiation électronique suivant la loi $i_{0} \sim t^{1,7}$. Après une irradiation de 10 minutes, correspondant à un flux intégré de $2 \times 10^{16}$ électrons $/ \mathrm{cm}^{2}$, $i_{0}$ commence à devenir indépendant du temps d'irradiation.

- Influence DE L'oxygène Sur LE COURANT D'OBSCURITÉ. - Le courant maximum d'obscurité qu'on peut atteindre sur nos cristaux varie selon l'échantillon entre $10^{-8}$ et $3 \times 10^{-7}$ A $\left(10^{-6}\right.$ et $3 \times 10^{-5} \mathrm{~A} / \mathrm{cm}^{2}$ ). Par exemple, un courant d'obscurité de $10^{-7}$ A a diminué de $20 \%$ au bout de 50 heures lorsque le cristal est maintenu sous vide et dans le noir ; lorsque le cristal est exposé à l'oxygène ou à l'air, il diminue plus rapidement. L'influence de l'oxygène sur ce courant d'obscurité $i_{0}$ est montrée sur la figure 4 qui représente la variation du courant d'obscurité en fonction du temps d'exposition à l'oxygène. Le courant diminue de 4 décades dans les 2 premières minutes qui suivent l'entrée du gaz. Ensuite, la diminution est plus lente et l'on atteint $10^{-13} \mathrm{~A}$ au bout de 8 heures sous oxygène. A ce moment, l'équilibre n'est pas atteint. En évacuant alors l'enceinte, le courant subit d'abord une faible diminution transitoire, ensuite il augmente de deux décades en deux heures. Une augmentation transitoire du courant est observée lorsque l'oxygène sec est à nouveau introduit (Fig. $4: 10 \mathrm{~h} \mathrm{30)}$. Ces variations transitoires sont d'autant plus importantes que le courant est plus faible.

L'échantillon est ensuite irradié à $5150 \AA$ A sous oxygène pendant deux heures. Après suppression de cet éclairement, la durée de la décroissance du courant est réduite à 20 heures par une série de quenching à $1,4 \mu \mathrm{m}$ non représentée sur la figure. A l'équilibre 


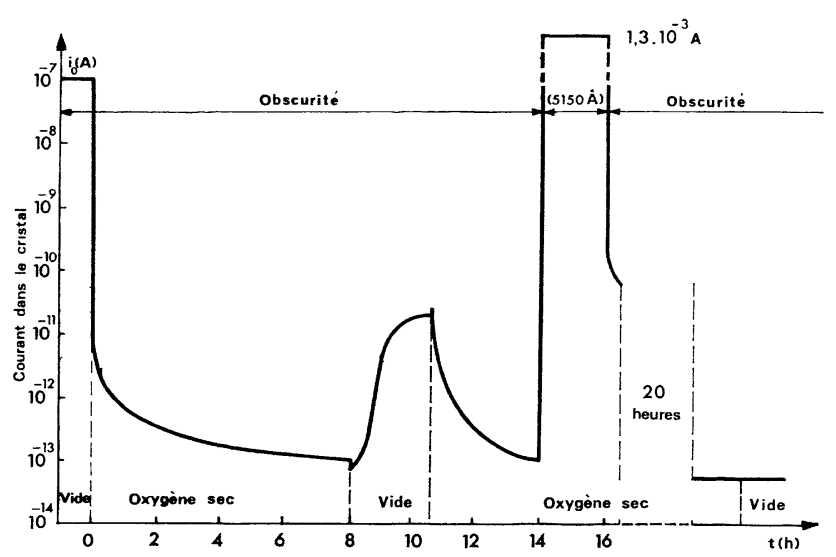

Fig. 4. - Evolution du courant d'obscurité en fonction du temps : la surface propre est d'abord exposée à l'oxygène à l'obscurité, placée sous vide, ensuite placée sous oxygène sous éclairement, enfin placée de nouveau sous vide à l'obscurité.

Dark current versus time : the surface is first exposed to oxygen in the dark, then to vacuum. Thereafter, the crystal surface is exposed to oxygen under light. Finally it is replaced into vacuum in the dark.

thermique, ou dans un état très proche de cet équilibre, le courant d'obscurité $\left(5 \times 10^{-14} \mathrm{~A}\right)$ n'augmente pas lorsque l'oxygène est évacué.
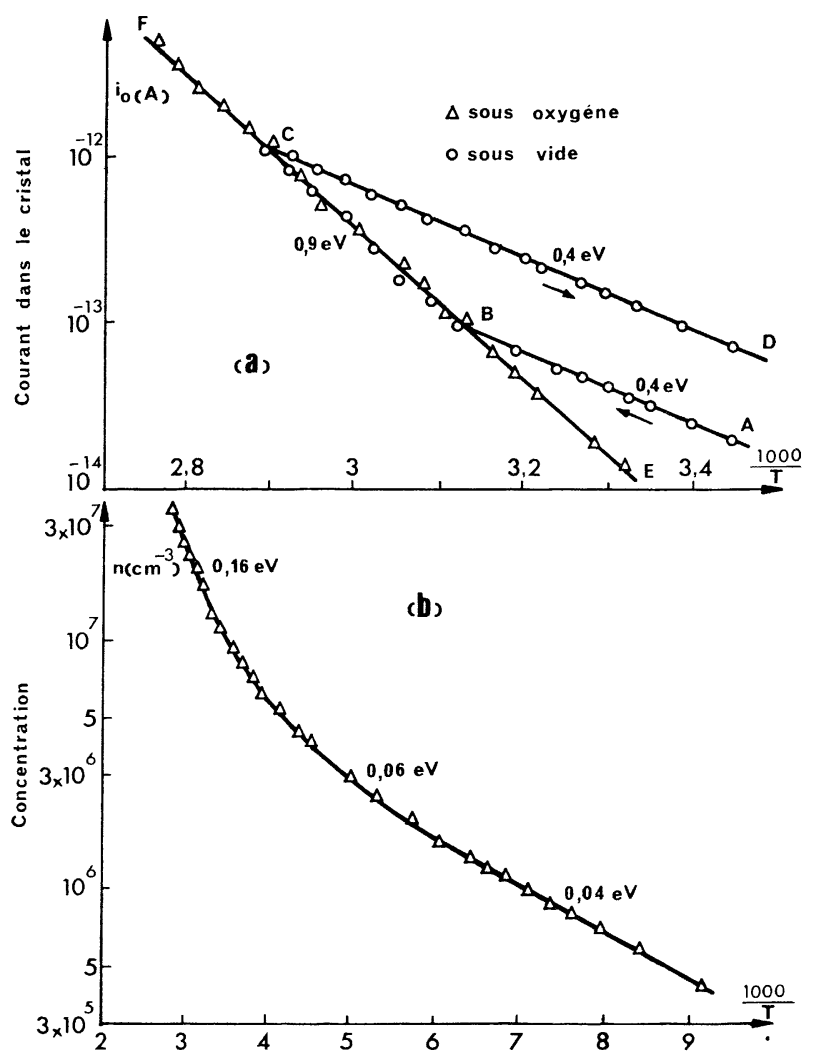

FIG. 5. $-a$ ) Courant d'obscurité en fonction de la température, le cristal se trouvant sous vide (0) et sous oxygène $(\Delta), b$ ) Concentration des électrons libres à l'obscurité en fonction de la température sur un cristal désorbé, sous vide.

a) Dark current versus temperature for a crystal in vacuum (0) and in oxygen $(\Delta) . b$ ) Free electron concentration in the dark versus temperature for a desorbed crystal in vacuum.
- VARIATION DU COURANT D'ObSCURITÉ EN FONCTION DE LA TEMPÉRATURE. - La figure 5 représente le courant d'obscurité $i_{0}$ en fonction de la température pour différents états d'adsorption. Lorsque l'échantillon se trouve dans une atmosphère d'oxygène sec (Fig. 5a), l'énergie d'activation déduite de la courbe est de $0,9 \mathrm{eV}$ durant l'échauffement et le refroidissement. Si l'échantillon est ensuite placé sous vide, on trouve successivement les énergies de 0,4 et $0,9 \mathrm{eV}$ en chauffant ; en refroidissant, seule l'énergie de $0,4 \mathrm{eV}$ est déterminée. Si le même cristal a subi une excitation électronique, de façon à faire remonter le courant à quelques $10^{-8} \mathrm{~A}$, on obtient la courbe de la figure $5 b$. Elle représente la concentration des électrons libres en fonction de la température calculée en supposant une variation de la mobilité suivant une loi en $T^{-3 / 2}$ et une valeur de $250 \mathrm{~cm}^{2} / \mathrm{V}$.s à $20^{\circ} \mathrm{C}$ [27]. Dans ce cas, on observe des énergies d'activation de 0,04, 0,06 et $0,16 \mathrm{eV}$.

Discussion. - Modèle DE CHIMISORPTION. - Les phénomènes observés peuvent être interprétés à partir du modèle de chimisorption de Williams et de Mark $[16,17,18]$.

L'oxygène est chimisorbé par piégeage des électrons libres du volume. Sur le cristal placé sous atmosphère d'oxygène, on trouve un niveau d'énergie à $0,9 \mathrm{eV}$ (Fig. 5a, courbe EFE réversible). Mark [28] a trouvé cette valeur pour des cristaux placés également sous oxygène. Sous vide, un deuxième niveau à $0,4 \mathrm{eV}$ est déterminé à partir de la courbe $5 \mathrm{a}$. Ces deux énergies de 0,4 et $0,9 \mathrm{eV}$ peuvent être attribuées respectivement à un niveau $\mathrm{O}^{--}$et un niveau $\mathrm{O}^{-}$. Des niveaux semblables ont été évalués sur le $\mathrm{ZnO}$ [14]. On pourrait envisager la chimisorption d'oxygène sous forme moléculaire également $[29,30]$. En outre, la nature exacte de la liaison entre l'oxygène et la surface ne peut être précisée ici. Bube a mis en évidence le niveau à $0,4 \mathrm{eV}$ à partir de la mesure de courants thermiquement stimulés [31]. Les niveaux à 0,41 et $0,83 \mathrm{eV}$ trouvés ensemble par Woods et Nicholas [22] sont interprétés dans le même sens par Bube.

- DÉSORPTION THERMIQUe - En chauffant sous vide un cristal adsorbé, ce sont d'abord les centres $\mathrm{O}^{--}$ qui perdent un électron pour devenir des centres $\mathrm{O}^{-}$: pente correspondant à $0,4 \mathrm{eV}$. Lorsqu'il n'y a plus de centres $\mathrm{O}^{--}$, les centres $\mathrm{O}^{-}$commencent à perdre leur électron (pente $0,9 \mathrm{eV}$ ). Les ions $\mathrm{O}^{-}$se transforment alors en oxygène physisorbé qui est libéré thermiquement. Pendant le chauffage sous vide, la concentration totale diminue donc. Au cours du refroidissement (CD) les électrons libres sont piégés par les ions $\mathrm{O}^{-}$pour former des niveaux $\mathrm{O}^{--}$: une énergie de $0,4 \mathrm{eV}$ est alors libérée. Par contre, sous oxygène, il y a toujours suffisamment d'atomes physisorbés disponibles pour permettre la formation de niveaux $\mathrm{O}^{-}$pendant le refroidissement (FE, pente $0,9 \mathrm{eV}$ ). La diminution de la concentration totale d'oxygène adsorbé peut être réalisée en élevant la température sous vide. 
- DÉSORPTION PAR BOMBARDEMENT ÉleCTRONIQUE - L'effet du bombardement électronique sur le courant d'obscurité est représenté en fonction de la durée d'irradiation sur la courbe c de la figure 3. A priori, le bombardement de la surface par des électrons entraîne une diminution de la concentration de l'oxygène adsorbé mais il reste à discuter dans quelle mesure, la création de lacunes de soufre formant des niveaux donneurs à la surface peut contribuer à l'augmentation du courant d'obscurité. Sébenne et al. [32, 33] ont trouvé qu'il suffit d'une énergie faible pour créer ces vacances de soufre à la surface du CdS. Ils ont observé un départ de soufre en chauffant sous vide un cristal à $400^{\circ} \mathrm{C}$. En bombardant sous vide des cristaux de CdS, Kulp et Kelley [34] ont constaté qu'une énergie aussi faible que $2,5 \mathrm{keV}$ est effectivement suffisante pour provoquer le départ de la surface d'atomes de soufre intersticiels. Par contre, il leur faut une énergie électronique supérieure à $115 \mathrm{keV}$ pour créer des vacances de soufre. Par ailleurs, la figure $5 b$ montre l'existence de plusieurs niveaux d'énergie. Or, on devrait s'attendre à trouver un seul niveau $(0,06 \mathrm{eV}$ [33]) s'il s'agissait de centres donneurs dus aux lacunes de soufre. En tenant compte de ces faits, et tant qu'il n'existe pas davantage d'expériences sur ce problème, nous concluons que la formation de lacunes de soufres pouvant intervenir dans le mécanisme de désorption est improbable.

$\mathrm{Au}$ cours de l'irradiation, la concentration des atomes chimisorbés diminue et la conductivité augmente suivant une loi $\sigma \sim t^{1,7}$ où $t$ est le temps. Pour préciser le mécanisme de la désorption, nous avons comparé les effets sur le courant d'obscurité d'une irradiation lumineuse pendant 12 heures à $4500 \AA$ avec ceux d'un bombardement électronique pendant 10 minutes. Dans les deux cas, le cristal se trouve initialement dans un état bien adsorbé $\left(i_{0}=5 \times 10^{-14} \mathrm{~A}\right)$ et l'intensité de la lumière est réglée de façon à observer un photocourant de $3 \times 10^{-4} \mathrm{~A}$ identique à celui obtenu sous un bombardement électronique de $5 \times 10^{-6} \mathrm{~A} / \mathrm{cm}^{2}$. Après l'éclairement, le courant d'obscurité reste très faible $\left(i_{0}=3 \times 10^{-13} \mathrm{~A}\right)$ tandis qu'après bombardement électronique il remonte à $3 \times 10^{-8} \mathrm{~A}$. La désorption par la lumière n'est donc pas efficace. La concentration des trous créés dans les deux cas étant la même, on peut déduire que leur recombinaison, avec les ions oxygène, est un processus peu probable. Shappir et Many [35] ont en effet trouvé une valeur très faible $\left(S_{p} \simeq 10^{-27} \mathrm{~cm}^{2}\right)$ pour la section efficace de capture $S_{p}$ des trous par les niveaux d'oxygène situés à $0,9 \mathrm{eV}$.

Sous irradiation électronique, la désorption est due à une collision des électrons avec les ions chimisorbés eux-mêmes. Tant que la concentration $\left[\mathrm{O}^{-}\right]$d'ions $\mathrm{O}^{-}$ ne varie pas notablement, la dencité $\Delta n$ des électrons libérés dans la bande de conduction à partir des ions $\mathrm{O}^{-}$, est proportionnelle au temps :

$$
\Delta n=C_{1}\left[\mathrm{O}^{-}\right] \Delta t .
$$

$C_{1}$ est une constante. Une pente supérieure à 1 (courbe $c$, Fig. 3) peut être obtenue si les ions $\mathrm{O}^{--}$interviennent aussi dans le processus de désorption. Lorsque les doses d'irradiation dépassent $2 \times 10^{16}$ électrons par $\mathrm{cm}^{2}$, la concentration des ions chimisorbés devient faible, le rendement de désorption diminue et le courant d'obscurité $i_{0}$ tend vers une valeur de saturation (courbe $c$, Fig. 3). Une dose de $10^{17}$ électrons $/ \mathrm{cm}^{2}$ est nécessaire pour la désorption optimale. Lorsque celle-ci est atteinte, le courant $i_{0}$ est dû aux donneurs peu profonds. Leurs niveaux d'énergie peuvent alors être déterminés (Fig. 5b). Ils varient d'un cristal à l'autre selon la nature du dopage.

- DÉSORPTION PAR POMPAGE. - La figure 4 prouve qu'il y a équilibre entre l'oxygène chimisorbé et l'oxygène physisorbé en surface. Lorsqu'on place sous vide un cristal qui a été exposé à l'oxygène dans le noir, le courant d'obscurité augmente. La diminution de la concentration de l'oxygène neutre entraîne celle de l'oxygène chimisorbé. Par contre, la figure 4 montre à droite que le courant d'obscurité n'augmente pas en faisant le vide à condition que l'adsorption ait été effectuée sous éclairement. Dans ces conditions, la concentration d'oxygène chimisorbé peut être supérieure à celle des donneurs peu profonds.

- BARRIÈre DE POTENTIEL. - Par ailleurs, il n'existe pas une barrière importante sur la surface du cristal : sur un échantillon adsorbé, aucune phototension n'est observée en éclairant celui-ci avec une lumière fortement adsorbée (4500 $\AA$ ). Sur des cristaux de $3 \times 10^{6} \mathrm{ohm} . \mathrm{cm}$ dans l'état adsorbé, Williams [16] n'observe pas de phototension : il en déduit que la concentration des états de surface chargés est très faible et qu'il n'y a pas de courbure des bandes. Dans notre cas, la variation relative du courant d'obscurité, présentée sur les figures 3,4 et 5 , est indépendante de la disposition des contacts utilisés (en sandwich ou en surface). On en déduit que le volume tout entier prend part au remplissage des pièges de surface.

Conclusion. - L'étude de la chimisorption sur un matériau de résistivité élevée montre qu'il suffit d'une concentration d'oxygène chimisorbé extrêmement faible pour rendre le matériau complètement isolant. En outre, on a mis en évidence l'avantage de l'irradiation électronique pour désorber complètement la surface. Ainsi, les niveaux donneurs deviennent accessibles à la mesure.

L'existence d'états de surface intrinsèques n'a pas été envisagée ; en fait, ces derniers jouent certainement un rôle peu important dans les cristaux ioniques ou semi-ioniques [29]. Many et Katzir [30] ont conclu qu'ils n'existaient pas sur la surface du CdS.

Remerciements. - Les auteurs remercient $\mathrm{Mr}$ le Professeur R. Guillien, M. G. Marchal pour leurs nombreuses suggestions concernant la rédaction du manuscrit, et Mr. C. Sebenne de la Faculté des Sciences de Paris pour de très fructueuses discussions. 


\section{Bibliographie}

[1] Reinhartz (K. K.) et Russel (V. A.), Solid-St. Electron., 1966, 9, 911.

[2] Waxman (A.), Solid-St. Electron., 1966, 9, 303.

[3] Dutton (R. W.) et Muller (R. S.), Solid-St. Electron., 1968, 11, 749.

[4] Faeth (P. A.), J. Electrochem. Soc., 1967, 114, 511.

[5] Capart (J. J.), ESTEC, Noordwijk, Hollande, communication privée.

[6] Geppert (D. V.), Cowley (A. M.) et Dore (B. V.), J. Appl. Phys., 1966, 37, 2458.

[7] Egorov (W. D.), Muller (G. O.) et Weber (H.), Phys. Stat. Sol., 1965, 12, 71.

[8] Mollwo (E.), Ann. Physik, 1948, 6, 230.

[9] Fritzsche (H.), Z. Physik, 1952, 133, 422.

[10] Ruppel (W.), Gerritsen (H. J.) et Rose (A.), Helv. Phys. Acta, 1957, 30, 495.

[11] Wolkenstein (Th.) et Karpenko (I. V.), J. Appl. Phys. Supp., 1962, 33, 460.

[12] Aigrain (P.) et Dugas (C.), Z. Elektrochem., 1952, $56,363$.

[13] Hauffe (K.) et EnGell (H. J.), Z. Elektrochem., 1952, $56,366$.

[14] Morrisson (S. R.), Advances in Catalysis, 1955, 7, 259.

[15] Melnick (D.A.), J. Chem. Physics, 1957, 26, 1136.

[16] Williams (R.), J. Phys. Chem. Solids, 1962, 23, 1057.

[17] Levine (J. D.) et Mark (P.), Phys. Rev., 1966, 144, 751.

[18] Micheletti (F. B.) et Mark (P.), J. Appl. Phys., 1968, 39. 5274.
[19] Weber (E. H.), Phys. Stat. Sol., 1968, 28, 649.

[20] Boer (K. W.), Physica, 1954, $20,1103$.

[21] Borchardt (W.), Phys. Stat. Sol., 1962, 2, 1575.

[22] Woods (J.) et Nicholas (K. H.), Brit. J. Appl. Phys., 1964, 15, 1361.

[23] Korsunskaya (N. E.), Markevich (I. V.) et SheinkMAN (M. K.), Phys. Stat. Sol., 1966, 13, 25.

[24] Korsunskaya (N. E.), Markevich (I. V.) et SheinkMAN (M. K.), 9e Int. Conf. on Physics of Semiconductors, Moscow, 1968, 1095.

[25] Kulp (B. A.) et Kelley (R. H.), J. Appl. Phys., 1961, 32, 1290.

[26] Stossel (W.) et Zimmermann (W.), Phys. Stat. Sol., $1968,30,311$.

[27] Spear (W. E.) et Mort (J.), Proc. Phys. Soc., 1963, $81,130$.

[28] Mark (P.), J. Phys. Chem. Solids, 1965, 26, 959.

[29] Krusemeyer (H. J.) et Thomas (D. G.), J. Phys. Chem. Solids, 1958, 4, 78.

[30] Many (A.) et Katzir (A.), Surface Sci., 1967, 6, 279.

[31] Bube (R. H.), Dussel (G. A.), Ching-Tao Ho et Miller (L. D.), J. Appl. Phys., 1966, 37, 21.

[32] Sebenne (C.), Thèse, Faculté des Sciences de l'Université de Paris, 1962.

[33] Pinchaux (R.), Sebenne (C.) et Balkanski (M.), the Structure and Chemistry of Solid Surfaces, John Wiley et Sons, Inc., 1969, 82-1.

[34] Kulp (B. A.) et Kelley (R. H.), J. Appl. Phys., 1960, 31, 1057.

[35] Shappir (J.) et Many (A.), Surface Sci., 1969, 14, 169. 